\title{
Enhanced optical nonlinearity of metasurfaces made of patterned graphene nanoribbons
}

\author{
Qun Ren, Jian Wei You and Nicolae C. Panoiu \\ Department of Electronic and Electrical Engineering, University College London, Torrington Place, London WC1E \\ 7JE, United Kingdom \\ qun.ren.15@ucl.ac.uk,j.you@ucl.ac.uk andn.panoiu@ucl.ac.uk
}

\begin{abstract}
We demonstrate that the effective nonlinear susceptibility of a graphene nanoribbon metasurface can be enhanced by 4 orders of magnitude as compared to that of a graphene sheet via a double-resonant plasmon excitation mechanism.
\end{abstract}

OCIS codes: $190.4223,190.4390,160.3918,050.6624,250.4390$.

\section{Introduction}

In this paper, we propose a linear and nonlinear homogenization approach for 2D graphene-based metasurfaces, and use it to demonstrate plasmon-induced enhancement of the effective second-harmonic (SH) susceptibility of such metasurfaces. In particular, we find that the second-harmonic generation (SHG) in graphene nanoribbon metasurfaces can be enhanced by orders of magnitude as compared to that of a graphene sheet [1] when surface plasmons are double-resonantly generated. Since the nonlinear susceptibility is a key physical quantity to describe nonlinear optical interactions, our results are particularly important for understanding nonlinear optical interactions in 2D materials.

\section{Results and Discussion}

The patterned graphene metasurface investigated in this paper, consisting of a 1D periodic distribution of graphene nanoribbons, and the corresponding unit cell are depicted in Fig. 1(a). The width of the nanoribbons is $w$ and throughout our analysis the period of the grating is $P_{x}=100 \mathrm{~nm}$, the chemical potential of graphene $\mu_{c}=0.2 \mathrm{eV}$, the relaxation time $\tau=0.1 \mathrm{ps}$, and the temperature $T=300 \mathrm{~K}$. The map of the absorption spectrum at the fundamental-frequency (FF), presented in Fig. 1(b), shows that the graphene grating supports a series of plasmon modes, seen as bright bands in this figure. More importantly, this figure illustrates the fact that for $w=65 \mathrm{~nm}$ the grating has plasmon modes both at the FF and SH. As we will demonstrate in what follows, the existence of this double plasmon resonance [2] leads to a significant enhancement of the SHG in the graphene metasurface.

This idea is illustrated in Fig. 2(a), where we present the spectra of the absorption, $A$, reflection, $R$, and transmission, $T$, calculated for the width $w=65 \mathrm{~nm}$ at which a double plasmon resonance occurs. In addition, we also show in this
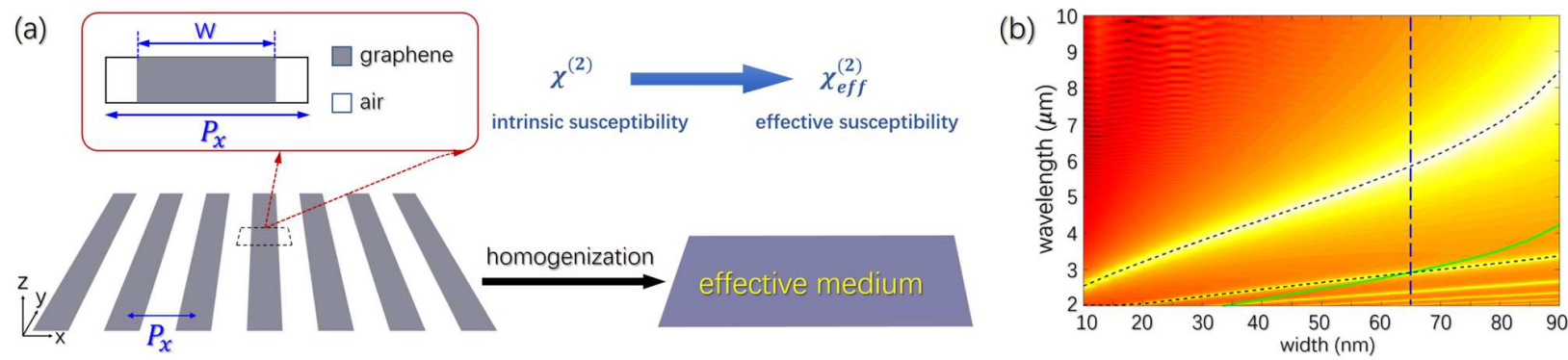

Fig. 1. (a) Schematics of a graphene-based metasurface, with period $P_{x}$ and width $w$, and the homogenization procedure used to calculate linear and nonlinear effective optical constants. (b) Map of absorption spectra at FF vs. the width of the graphene nanoribbons. The dashed curves mark the plasmon resonance modes, the green line indicates the half-wavelength of the fundamental plasmon, and the vertical dashed line the width at which the double-resonant SHG enhancement occurs. 
same figure the $\mathrm{SH}$ spectrum, determined for the same value of the width. This figure suggests that, firstly, $A, R$, and $T$ have a series of resonances occurring at common wavelengths. More importantly, however, one can see from these plots that indeed, for this width, the wavelength of the fundamental plasmon, $\lambda=5.84 \mu \mathrm{m}$, is twice as large as that of the second-order plasmon. This means that the incident optical field is strongly enhanced at the FF, due to the existence of the fundamental plasmon, and the nonlinear optical field at the $\mathrm{SH}$ is efficiently radiated in the continuum, due to the existence of the second-order plasmon at the $\mathrm{SH}$ wavelength. As a result, extremely strong nonlinear optical response of the graphene grating is achieved.
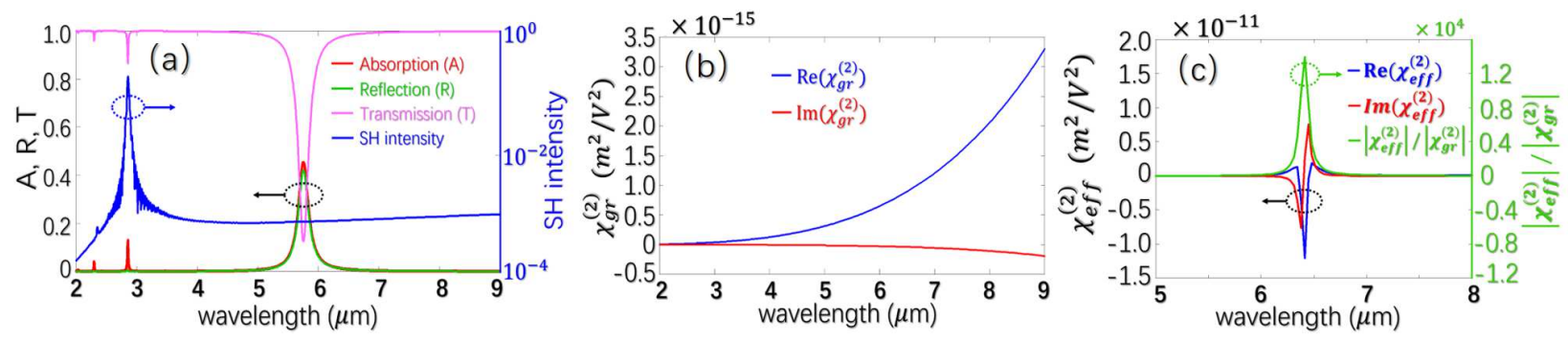

Fig. 2. (a) Spectra of absorption, reflectance, transmittance, and SH intensity. (b) Wavelength dependence of SH susceptibility of graphene. (c) Wavelength dependence of effective SH susceptibility and enhancement of effective SH susceptibility.

In order to quantify the enhancement of the SHG in the graphene metasurface, we compare the effective secondorder susceptibility of this metasurface, $\chi_{\mathrm{eff}}^{(2)}(\Omega ; \omega)$, where $\Omega=2 \omega$ is the SH frequency, with that of a uniform graphene sheet. The latter is plotted in Fig. 2(b), and is given by [3]: $\chi_{\mathrm{gr}}^{(2)}(\Omega ; \omega)=-\left[i /\left(\varepsilon_{0} \Omega h\right)\right] \sigma^{(2)}(\Omega ; \omega)$, where $h$ is the thickness of graphene and $\sigma^{(2)}(\Omega ; \omega)=3 i e^{3} v^{2} /\left[8 \pi \hbar^{2}\left(\omega+i \tau^{-1}\right)^{3}\right]$ is the SH conductivity of graphene [4]. Moreover, the nonlinear polarization at the $\mathrm{SH}$ can be determined from the electrical field at the FF via $\left[P_{i}\right]_{3 \times 1}=\left[\chi_{\mathrm{gr}, i j k}^{(2)}\right]_{3 \times 3^{2}}\left[E_{j} E_{k}\right]_{3^{2} \times 1}$, where $\chi_{\mathrm{gr}, i j k}^{(2)}$ is the second-order susceptibility of graphene. Finally, the effective nonlinear susceptibility of the graphene metasurface is determined as $\chi_{\mathrm{eff}, i j k}^{(2)}(\Omega ; \omega)=q_{\mathrm{eff}, i j k} /\left(E_{\mathrm{eff}, j} E_{\mathrm{eff}, k}\right)$, where $q_{\mathrm{eff}, i j k}(\Omega)=\int \chi_{\mathrm{gr}, i j k}^{(2)}(\Omega ; \omega, \mathbf{r}) E_{j}(\omega, \mathbf{r}) E_{k}(\omega, \mathbf{r}) \mathbf{d r} / V$ and $E_{\mathrm{eff}, i}$ is the $i$ th component of the averaged field at the FF.

The main results of these calculations are summarized in Fig. 2(c), where the dominant component of the effective nonlinear susceptibility, $\chi_{\text {eff,iii }}^{(2)}(\Omega ; \omega)$, is presented. It can be observed in this figure that, because of the doubleresonance feature of the graphene grating [2], the effective second-order susceptibility is enhanced by more than 4 orders of magnitude as compared to the intrinsic nonlinear susceptibility of graphene, at the double-resonance wavelength. This remarkable enhancement could be important in practical applications, especially for designing a large and tunable nonlinear susceptibility of $2 \mathrm{D}$ photonic nanodevices at predesigned operation frequencies.

\section{Conclusion}

In summary, by homogenizing the nonlinear susceptibility of a graphene metasurface, we demonstrate that its nonlinear optical response is enhanced by more than four orders of magnitude as compared to that of a uniform graphene sheet. We also identified the underlying physical mechanism responsible for this enhancement, namely the resonant excitation of plasmons both at the fundamental frequency and second-harmonic.

\section{References}

1. J. J. Dean and H. M. van Driel, "Second harmonic generation from graphene and graphitic films," Appl. Phys. Lett. 95, 261910 (2009).

2. J. W. You, J. You, M. Weismann and N. C. Panoiu, "Double-resonant enhancement of third-harmonic generation in graphene nanostructures,” Phil. Trans. R. Soc. A 375, 20160313 (2017).

3. Y. Q. An, J. E. Rowe, D. B. Dougherty, J. U. Lee and A. C. Diebold, "Optical second-harmonic generation induced by electric current in graphene on Si and SiC substrates," Phys. Rev. B 89, 115310 (2014).

4. J. D. Cox, I. Silveiro and F. J. García de Abajo, "Quantum effects in the nonlinear response of graphene plasmons," ACS Nano 10, 1995-2003 (2016). 